\title{
Investigating and Comparing Two Different CAD Methodologies to Create Top-down Assemblies
}

\section{Dr. Ibrahim F. Zeid, Northeastern University}

Ibrahim Zaid is a professor of mechanical, industrial, and manufacturing engineering at Northeastern University. He received his Ph.D. degree from the University of Akron. Zeid has an international background. He received his B.S. (with highest honor) and M.S. from Cairo University in Egypt. He has received various honors and awards both in Egypt and the United States. He is the recipient of both the Northeastern Excellence in Teaching Award and the SAE Ralph R. Teetor National Educational Award. 


\title{
INVESTIGATING AND COMPARING TWO DIFFERENT CAD METHODS TO CREATE TOP-DOWN ASSEMBLIES
}

\begin{abstract}
There are two main approaches to create CAD assemblies using commercial CAD/CAM systems: bottom-up or top-down. The bottom-up approach is the traditional approach. There is only one method to follow to create assemblies using the bottom-up approach: create parts, and then assemble them. The top-down approach is more efficient to use than the bottom-up for large and complex assemblies because it reduces errors within the assembly. There are two methods to create assemblies using the top-down approach: layout sketch, master model. These two methods are vastly different. Their technical comparisons and evaluations are not available. This paper investigates the merits, strengths, and weaknesses of each method. We have designed three case studies to aid in our research study. The three case studies involve three assemblies: simple, intermediate, and complex. The four differentiating criteria of the three assembly models are the number of parts per assembly, the number of sketch planes required to create assembly parts, total number of feature required to create all assembly part, and the number and type of features required to create a given part. The pencil represents a simple assembly. The hinge represents an intermediate assembly. And the gearbox represents a complex assembly. Utilizing a commercial CAD/CAM system (SolidWorks), we have created each assembly using each of the two methods. Based on our research results, we conclude that the master model method is the most robust, flexible, and adaptable method to use for the top-down approach.
\end{abstract}

Keywords: Assemblies; Top-down approach; Bottom-Up approach; Layout; Master model, CAD/CAM, SolidWorks.

\section{INTRODUCTION}

In the early days before the CAD/CAM field evolved, standard product development process involved part manufacturing followed by the physical assembly of the components to create the product. This approach was risky as there is no assurance of successfully achieving the best product development. After introduction of CAD/CAM, CAD/CAM systems such as SolidWorks and Creo provide product simulation, synthesis, and verification [1]. CAD/CAM systems enable designers to create parts and assemble them to test their design concepts, all virtually before production [2 - 4].

There are two main approaches to create CAD assemblies using commercial CAD/CAM systems: bottom-up or top-down. The bottom-up approach [4] is more intuitive and therefore more commonly used. This is the traditional approach. We follow three steps to create assemblies using the bottom-up approach: (1) create the parts, (2) insert them into an assembly model, and (3) use mates to assemble the parts. This approach is more useful for small assemblies consisting of hundred or thousand components [5].

The top-down approach [5] is also known as in-context approach. It is preferred for conceptual design when a design team is trying to conceive a new product and the layout of its components relative to each other in the assembly. It is a first step to define the design intent of the product (assembly) being designed. The top-down approach is also more efficient to use than the bottom-up for large and complicated assemblies because it reduces errors within the assembly. Some view the top-down approach as a way to claim space for the components in the assembly because it shows how the components are laid relative to each other. Others view it as a way to establish relationships and parameters for the components of the assembly and their dimensions. This approach is more useful for large and complex assemblies [5].

One of the problems with top-down assembly approach is to find the best method to create the assembly. There are two methods to create top-down assemblies: layout sketch and master model. Technical comparisons and evaluations of these two methods are not available. Also, some CAD/CAM systems are more robust than others in implementing these methods as discussed in this paper.

This paper investigates the two methods for the top-down assembly approach. The paper investigates the merits, strengths, and weaknesses of each method. We have designed three case studies to aid in our research study. The three case studies involve three assemblies: simple, intermediate, and complex. The paper identifies and discusses the differentiating criteria of the three assembly models. The pencil represents a simple assembly. The hinge represents an intermediate assembly. And the gearbox represents a complex assembly. Utilizing a commercial CAD/CAM system (SolidWorks in this paper), we have created each assembly using each of the two methods and provide detailed analysis and conclusion in this paper.

\section{LAYOUT SKETCH METHOD}


The layout of sketch aids the CAD designer to understand and investigate the relative placements of assembly components and their clearance margins. It is also useful to define the space utilization by individual component, skeleton model and relation between components. Thus, it enables the CAD designer to manage assembly requirements effectively in one sketch [5].

Different CAD/CAM systems implement the layout sketch method differently. For example, SolidWorks provides the layout sketch as part of its assembly module [6]. Creo, on the other hand, provides the layout sketch both as a separate module and as part of its assembly module [7]. This paper focuses on SolidWorks only. In solidworks, the layout sketch has one orientation in the 3D modeling space, the front sketch plane. That introduces severe limitations to create assemblies, especially for complex assemblies.

SolidWorks layout sketch method uses the concept of "block" to aid in creating assemblies. The designer groups entities after creating them into blocks. Each block defines an assembly component (part) of the assembly. For example, if an assembly consists of two parts, the designer needs to create two blocks, each defining a part. A block behaves as a single entity. Once a block is defined, the designer uses it to create its corresponding part. The blocks are restricting in that the entities belonging to one block cannot be used to create another block.

In order to create an assembly with layout sketch method in SolidWorks, we typically follow these steps:

1. Start SolidWorks

2. Select assembly mode

3. Select the layout sketch method

4. Start sketching and dimensioning the required entities in the layout sketch plane (front plane)

5. Create necessary blocks by grouping the entities

6. Convert blocks into parts

7. Save the parts in external files

8. $\quad$ Open each part external file and create the part feature(s)

9. Create appropriate mates between parts

\section{MASTER MODEL METHOD}

The core idea behind the master model method $[8,9]$ is to create all the assembly parts in one part file (master or parent part) and then spin them off (split them) into multiple child parts. As a result, the master model method creates a parent/child relationship more effectively in 3D modeling. For example, when the CAD designer makes changes in the parent part, the changes are automatically applied to the child part. In general, it enables the designer to make changes in assembly easily. For more complex assembly it also allows user to understand all the clearance, tolerances and space claim concepts.

CAD/CAM systems implement the concept of master model differently. In SolidWorks, the mater model is created as a part at the part level. In Creo, it is created as an assembly at the assembly level. In SolidWorks, we create the master part the same we create a single part. The only difference is when creating multiple features, we do not merge the features together; we keep them as separate bodies, i.e. we create a disjoint solid. We save these bodies into separate external files and assemble them to create the assembly.

In order to create an assembly with master model method in SolidWorks, we typically follow these steps:

1. Start SolidWorks

2. Select the part mode

3. Create master part features (separate solid bodies)

4. Save the parts in external files

5. Open a new assembly file

6. Assemble the externally saved parts by inserting them into assembly model and applying the required mates

We have selected three assemblies to create using both methods of the layout sketch approach: simple assembly (a pencil), an intermediate one (a door hinge), and a complex one (a gearbox). The selection criteria are discussed in the "Analysis and Discussion" section of the paper. The required number of sketch planes to create assembly parts is the most decisive criterion in determining the complexity level of creating assemblies using the top-down approach. The goal of creating these three assemblies is to evaluate the scalability and robustness of the two methods: layout sketch and master model.

\section{PENCIL: LAYOUT SKETCH METHOD}


The pencil is the simple assembly. It has four parts: body, lead, cap, and eraser. We create the sketch for each part in the layout sketch plane (front plane). Thus, we have to think in advance of how to create each part using the front sketch plane only. In the pencil case, we create the four parts as revolves (axisymmetric features). The steps used to create the pencil assembly are listed below:

1. Create the body part: after activating the layout sketch in SolidWorks, we create the body sketch shown in Figure 1. Next, we create a block using the body sketch, save the resulting part externally, open it, revolve it to create the pencil body shown in Figure 2.

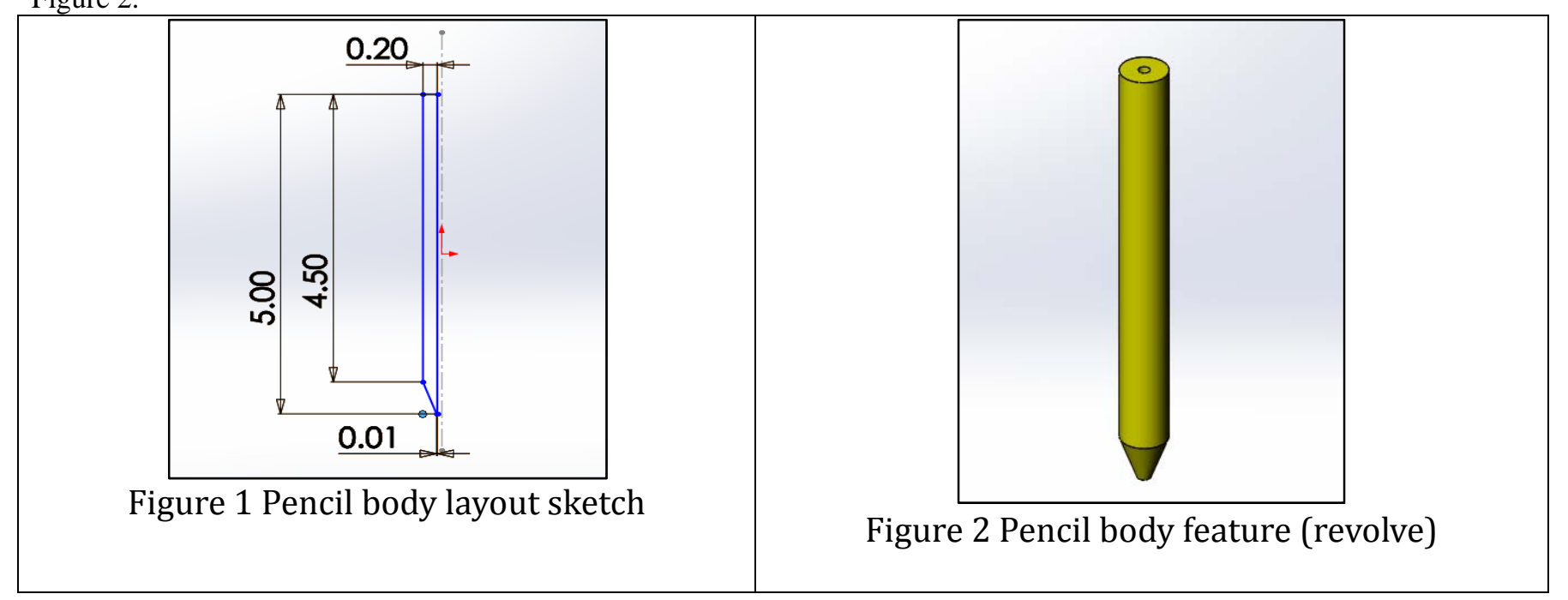

2. Create the lead part: follow the same steps as for the pencil body to create the lead sketch (Figure 3) and the lead feature (Figure 4).

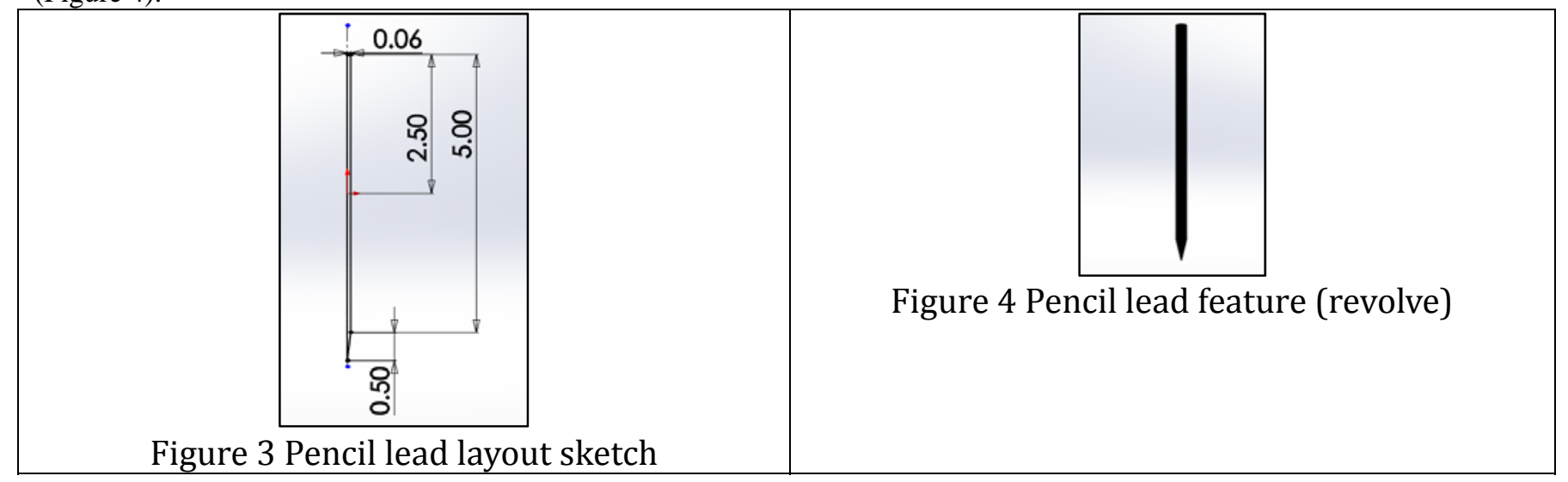

3. Create the cap part: follow the same steps as for the pencil body to create the cap sketch (Figure 5) and the cap feature (Figure 6).

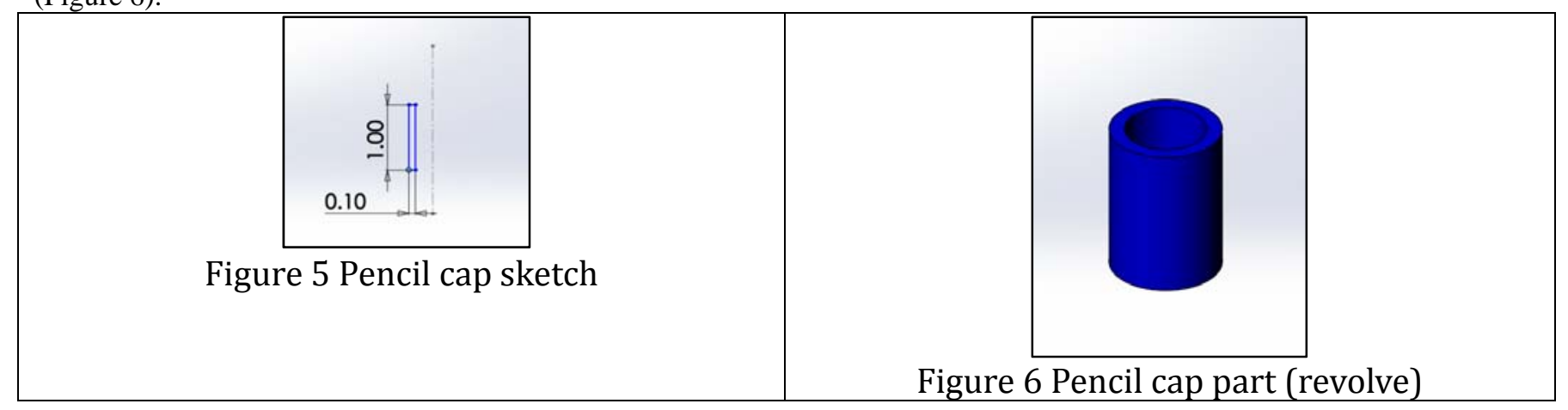


4. Create the eraser part: follow the same steps as for the pencil body to create the eraser sketch (Figure 7) and the eraser feature (Figure 8).

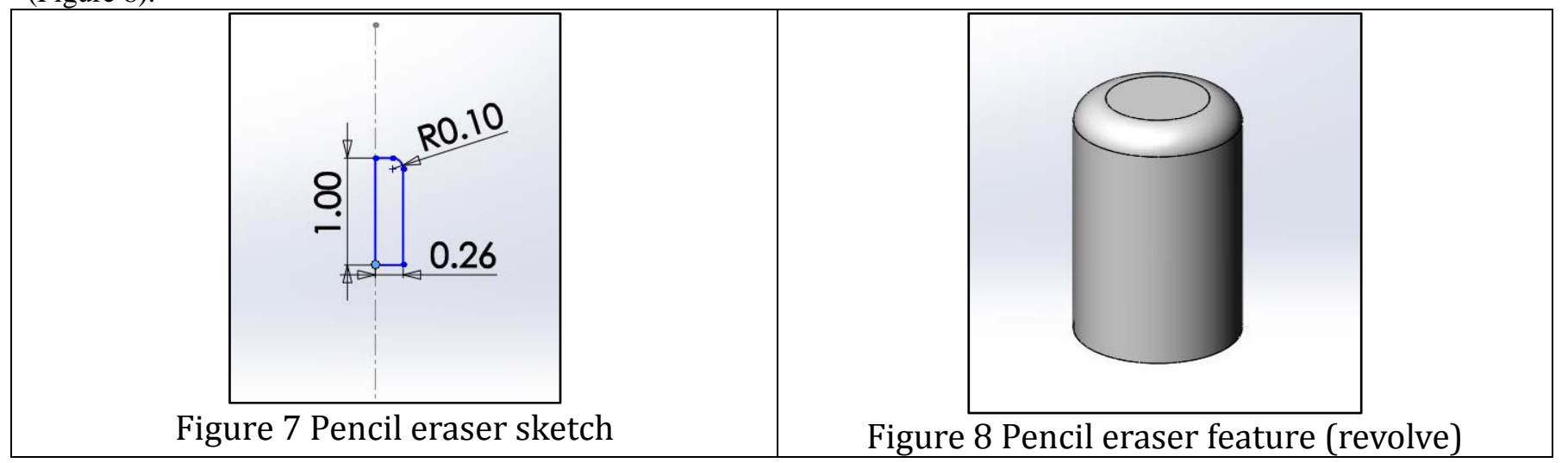

The final pencil assembly is show in Figure 9 and Figure 10 shows the exploded view. The assembly, at this stage, does not have assembly mates between its four individual parts. But they can be added if needed. The pencil parts are shown assembled because they are created at their layout sketch locations. That is the very core point of using the layout sketch.

\begin{tabular}{|c|c|}
\hline Figure 9 Complete pencil assembly (Layout \\
method)
\end{tabular}

\section{PENCIL: MASTER MODEL METHOD}

Creating the pencil using the master model method is much simpler and more straightforward than using the layout sketch method. We create the four parts of the pencil in one SolidWorks part. The key is to turn off the "Merge Results" function in SolidWorks. We still create the four parts as revolves as we did in the layout sketch method. The steps used to create the pencil assembly are listed below:

1. Create the body feature: after opening a new part, create the body sketch shown in Figure 1 . Note that the body sketch geometry is the same regardless of using the layout sketch or the master model method. Revolve the sketch to create the pencil body feature (same as shown in Figure 2).

2. Create the pencil lead feature: create the lead sketch shown in Figure 3. Revolve the sketch to create the pencil lead feature (same as shown in Figure 4).

3. Create the pencil cap: create the cap sketch shown in Figure 5. Revolve the sketch to create the pencil cap feature (same as shown in Figure 6).

4. Create the pencil eraser: create the eraser sketch shown in Figure 7. Revolve the sketch to create the pencil eraser feature (same as shown in Figure 8). 
5. Save the four parts externally: we need to spin off the pencil four parts by saving them as external parts in preparation to create the pencil assembly. We simply right click each solid body in the current part features tree and save it in a file or save all the solid bodies by using "save bodies" option in right click menu.

6. Assemble the parts to create the pencil shown in figure 11. This is the same step as in the layout sketch method. Figure 9 and 11 are identical as expected.

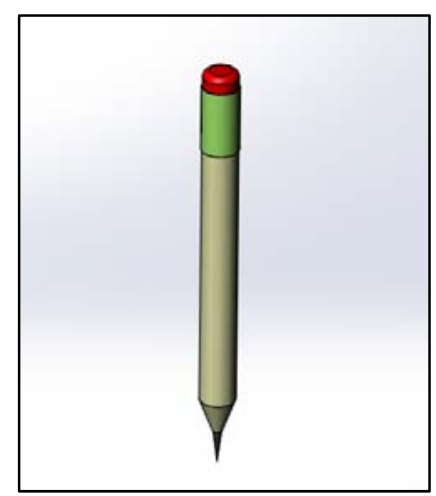

Figure 11 Complete pencil Assembly (Master model method)

After creating the pencil assembly using both methods, we can conclude that the layout sketch method requires more steps to create the assembly; layout sketch requires creating blocks. Moreover, there are lots of other challenges that need in-depth attention in order to reduce the total modeling time.

\section{HINGE: LAYOUT SKETCH METHOD}

The hinge is an intermediate assembly. It has three parts: plate -1 , plate-2 and pin. We create the sketch for each part in the layout sketch plane (front plane). Thus, we have to think in advance of how to create each part using the front sketch plane only. In the hinge case, we create the three parts as a combination of extrusions and extrusion cuts. The steps used to create the hinge assembly are listed below:

1. Create plate-1 part: after activating the layout sketch in SolidWorks, create the plate-1 sketch shown in Figure 12 . Next, we create a block using the plate-1 sketch, save the resulting part externally, open it, extrude it and perform other necessary features to create the hinge plate-1 shown in Figure 13.

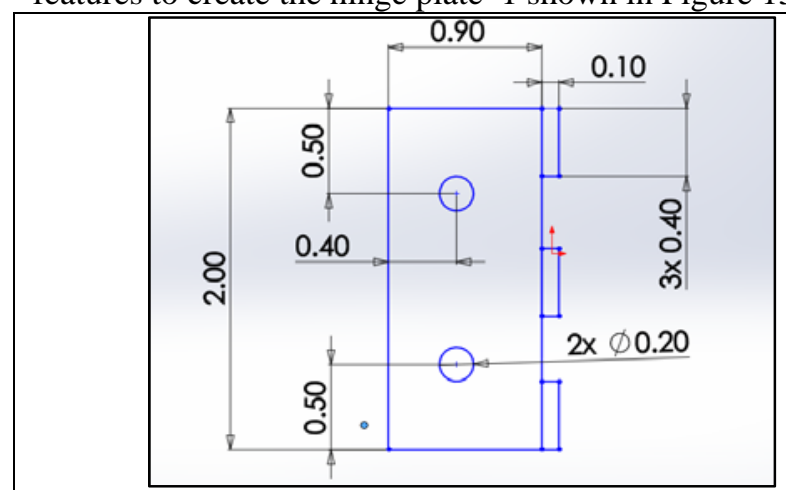

Figure 12 Hinge plate-1 layout sketch

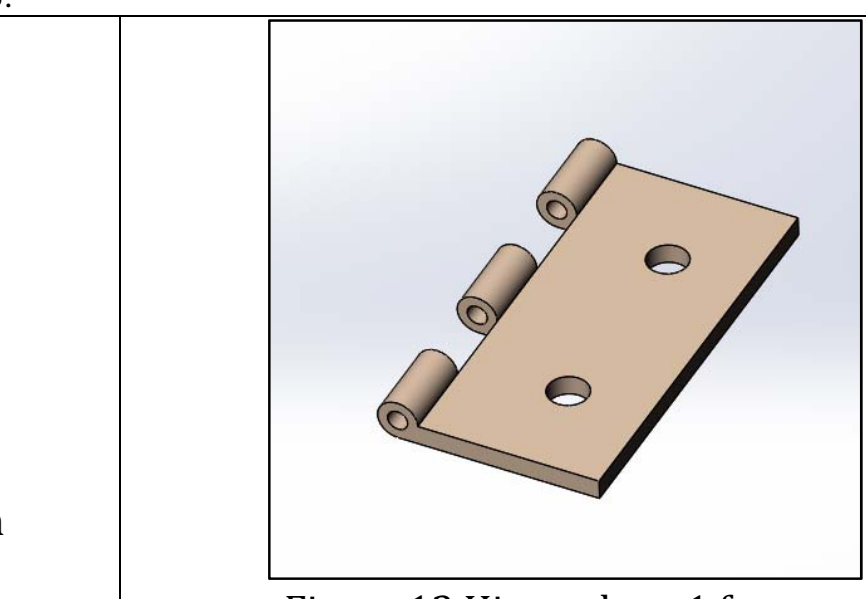

Figure 13 Hinge plate-1 feature 
2. Create the plate-2 part: follow the same steps as for the plate-1 to create the plate-2 sketch (Figure 14) and the plate - 2 feature (Figure 15).

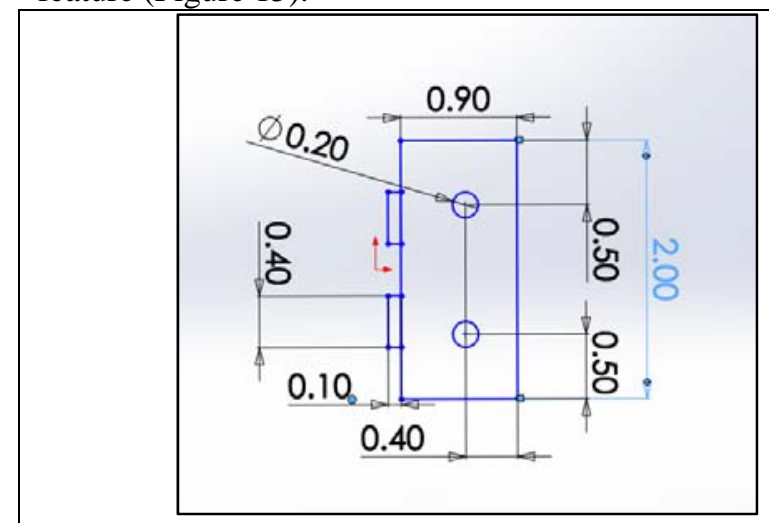

Figure 14 Hinge plate-2 layout sketch

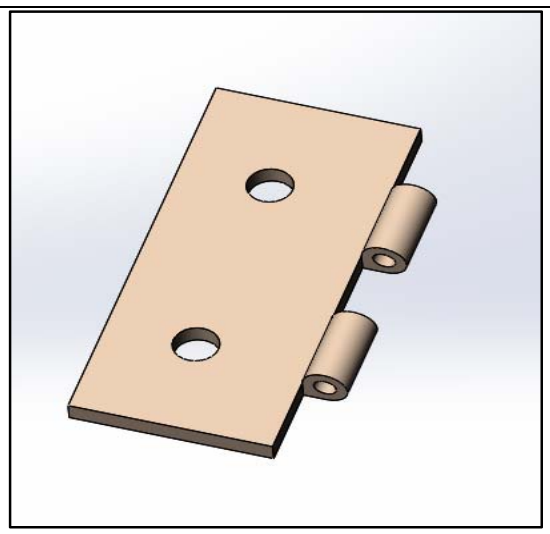

Figure 15 Hinge plate-2 feature

3. Create the pin part: follow the same steps as for the plates to create the pin sketch (Figure 16) and the pin feature with revolve (Figure 17).

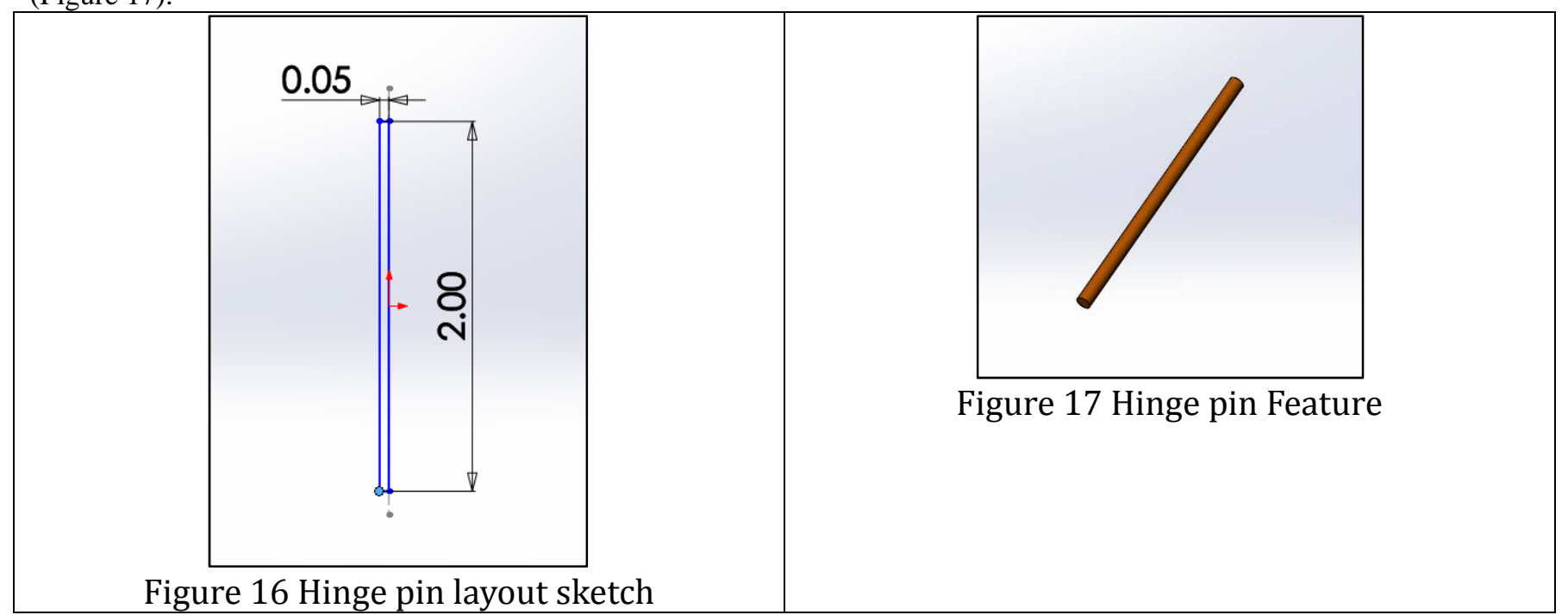

The final hinge assembly is show in Figure 18 and Figure 19 shows the exploded view. The assembly, at this stage, does not have assembly mates between its three individual parts. But they can be added if needed. The hinge parts are shown assembled because they are created at their layout sketch locations.

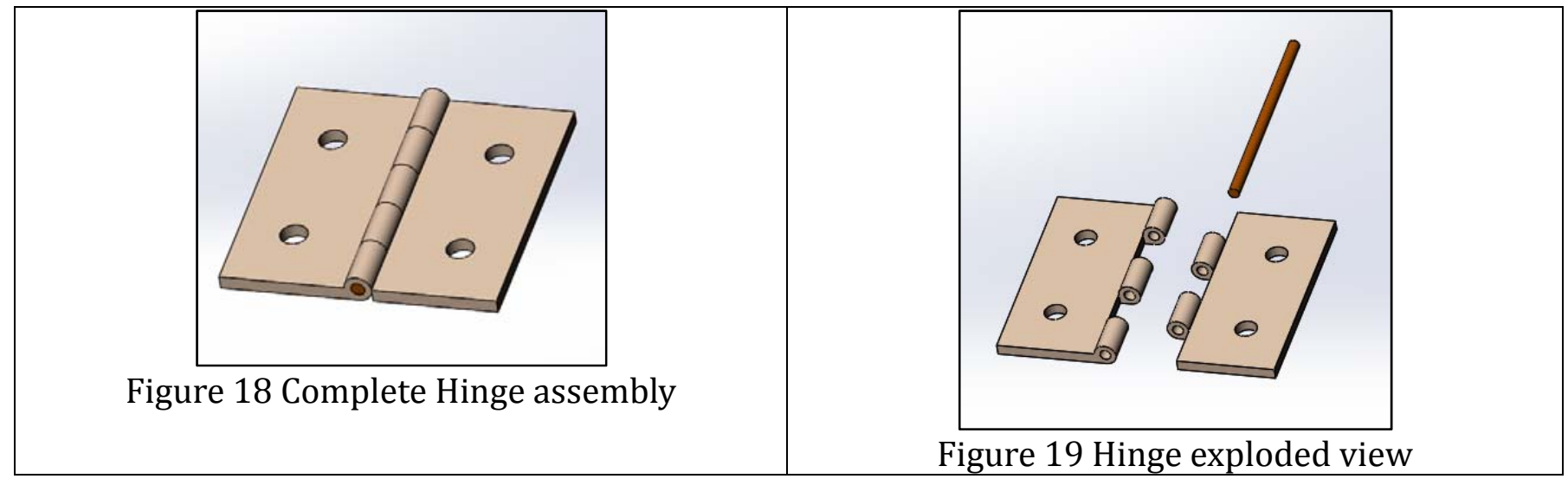




\section{HINGE: MASTER MODEL METHOD}

Creating the hinge using the master model method is much simpler and more straightforward than using the layout sketch method. We create the three parts of the hinge in one SolidWorks parts. The key is to turn off the "Merge Results" function in SolidWorks and use the "Feature Scope" function which allows the designer to choose parts to merge with each other if needed. We still create the three parts as extrusion as we did in the layout sketch method. The steps used to create the hinge assembly are listed below:

1. Create plate-1 feature: after opening a new part, create the plate-1 sketch shown in Figure 12 . Extrude the sketch and perform other features to create the hinge plate-1 feature (same as shown in Figure 13).

2. Create plate-2 feature: create the side plate sketch to provide distance required between two plates and mate the features for pin holding as shown in Figure 20. Extrude the sketch and perform other features required to create the plate-2 feature (same as shown in Figure 15).

3. Create the pin: create the pin sketch shown in Figure 21. Extrude the sketch to create the pin feature (same as shown in Figure 17).

\begin{tabular}{|l}
\hline $\mid$ \\
Figure 20 Hinge plate-2 sketch
\end{tabular}

4. Save the four parts externally: we need to spin off the hinge three parts by saving them as external parts in preparation to create the pencil assembly. We follow the same steps we used for the pencil master model method.

The final hinge assembly is the same as shown in Figures 18 and 19. Similar to the layout sketch method, the assembly, at this stage, does not have assembly mates between its three individual parts. But they can be added if needed. The hinge parts are shown assembled because they are created at their proper sketch locations.

One can see that as complexity of model increases that limits the layout sketch method and forces designer to represent the all features of component into 2D plane (layout plane/ front plane). While designer can create the same features of same component more easily in master model. Layout model also required more steps to create same hinge model than master model.

\section{GEARBOX: LAYOUT SKETCH METHOD}

The gearbox is a complex assembly. It has seven parts: housing, 4 spur gears, shaft and connector pin. We create the sketch for each part in the layout sketch plane (front plane). Thus, we have to think in advance of how to create each part using the front sketch plane only. In the gearbox case, we create the seven parts as a combination of extrusion and other features. The steps used to create the gearbox assembly are listed below:

1. Create the housing part: after activating the layout sketch in SolidWorks, create the housing sketch shown in Figure 22. Next, we create a block using the housing sketch, save the resulting part externally, open it, perform necessary features required to create the gearbox housing shown in Figure 23. 


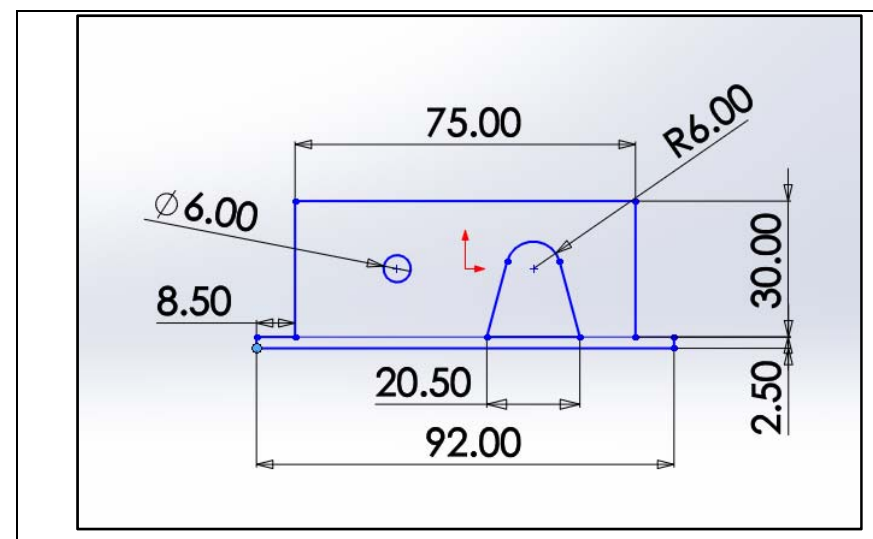

Figure 22 Gearbox housing layout sketch

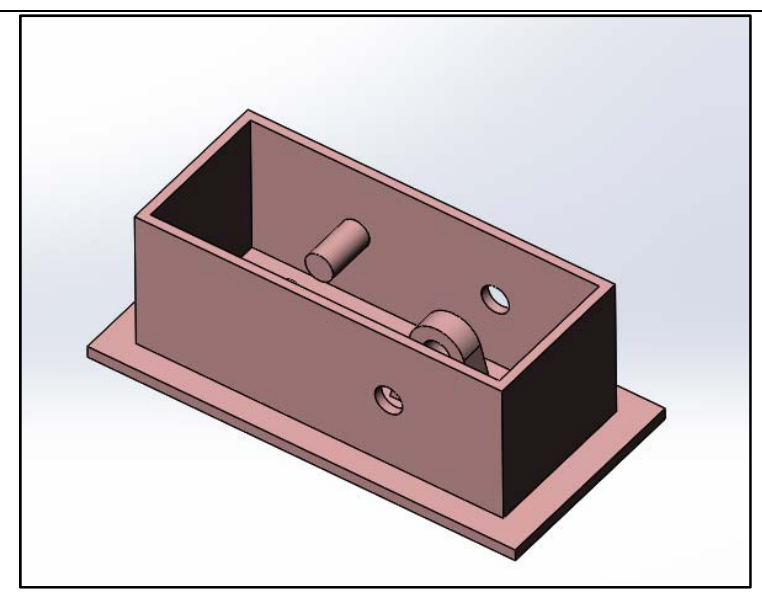

Figure 23 Gearbox housing feature

2. Create the two large spur gears: follow the same steps as for the gearbox housing to create the large gear sketch (Figure 24) and the large gear feature (Figure 25).

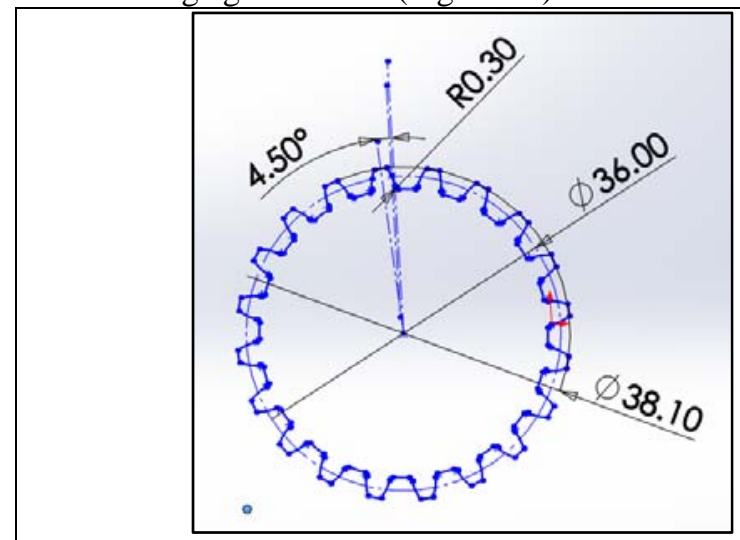

Figure 24 Gearbox large gear layout sketch

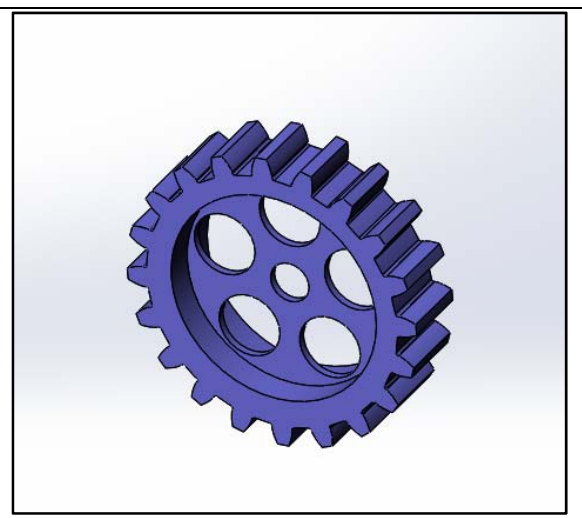

Figure 25 Gearbox large gear feature

3. Create the two small spur gears: follow the same steps as for the large gear to create the small gear sketch (Figure 25) and the small gear feature (Figure 26).

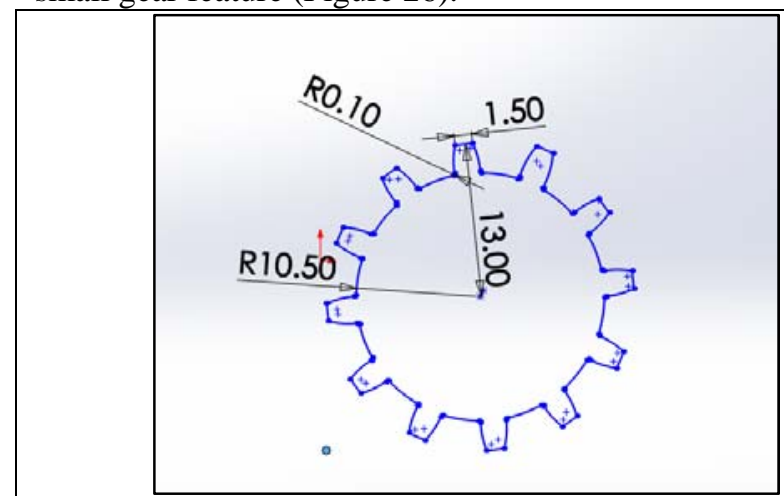

Figure 26 Gearbox small gear layout sketch

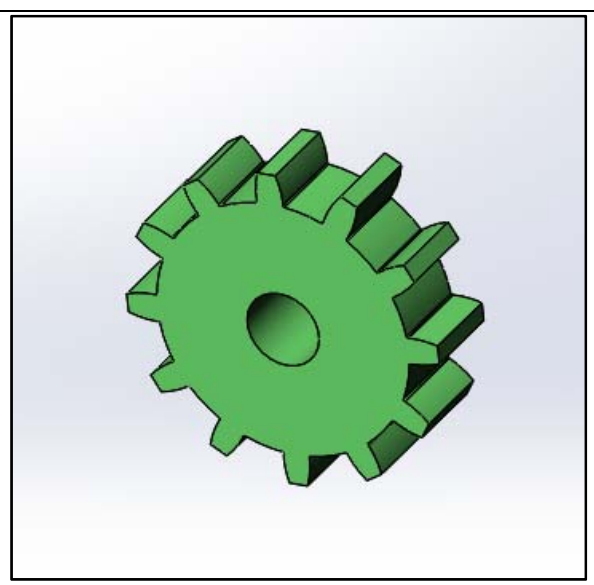

Figure 27 Gearbox small gear feature

4. Create the shaft part: follow the same steps as for the gears to create the shaft sketch (Figure 28) and the shaft feature (Figure 29). 


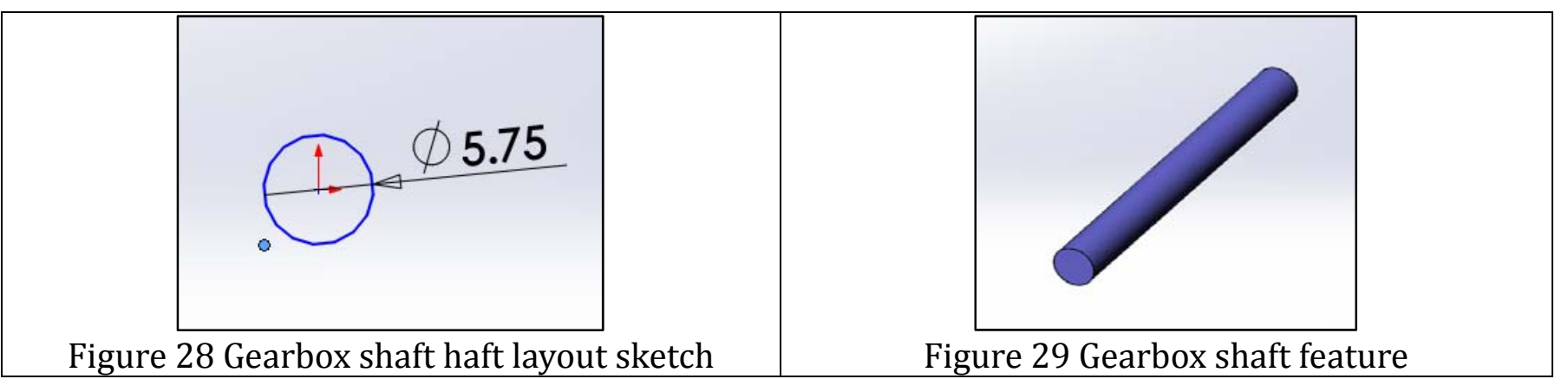

5. Create the connector part: follow the same steps as for the gearbox shaft to create the connector sketch (Figure 30) and the connector feature (Figure 31).

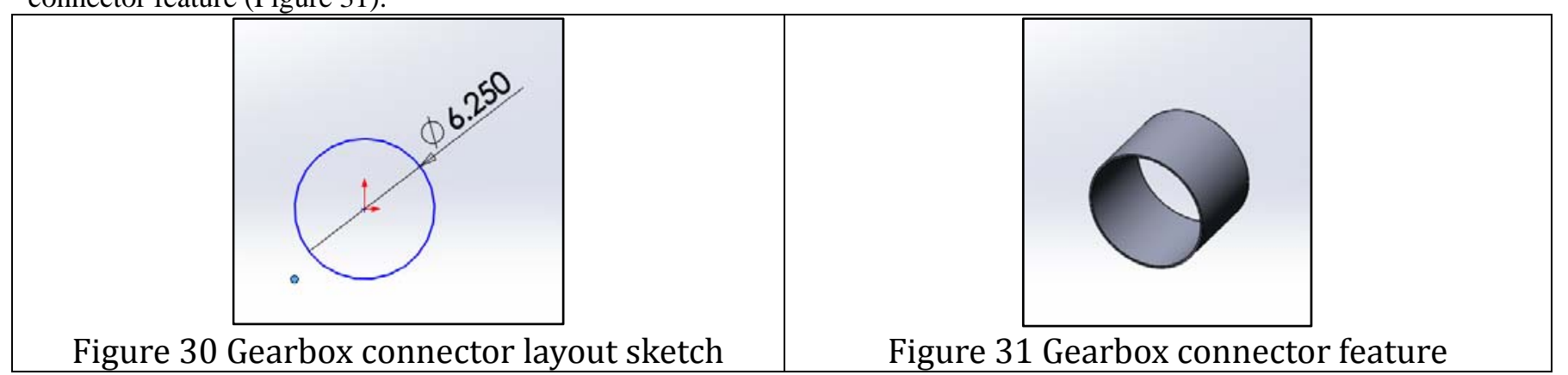

The final gearbox assembly is shown in Figure 32 and Figure 33. The assembly, at this stage, does not have assembly mates between its seven individual parts. But they can be added if needed. The gearbox parts are shown assembled because they are created at their layout sketch locations.

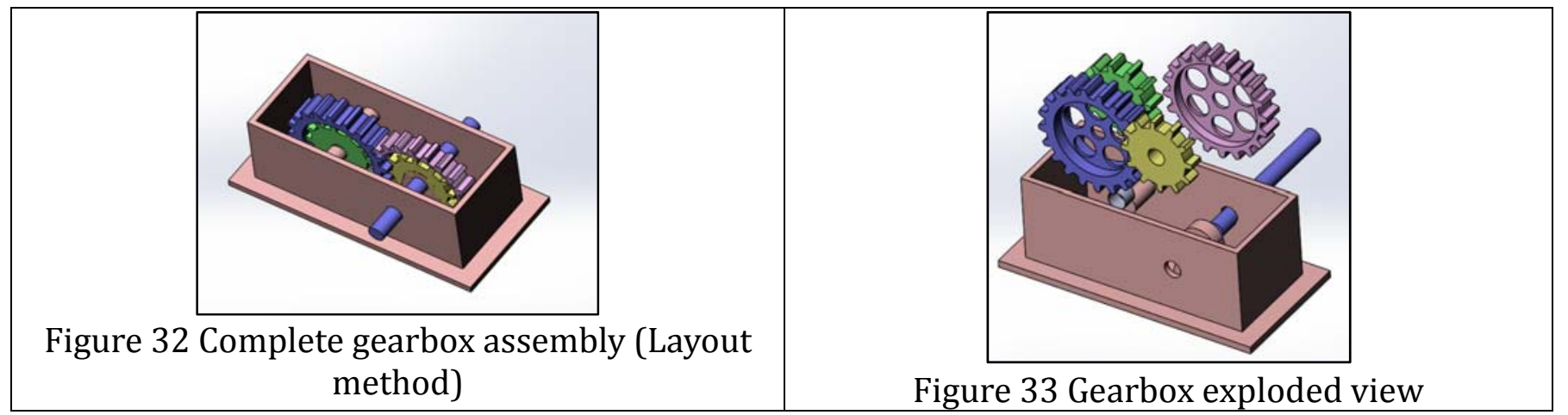

\section{GEARBOX: MASTER MODEL METHOD}

Creating the gearbox using the master model method is much simpler and more straightforward than using the layout sketch method. We create the seven parts of the hinge in one SolidWorks parts. The key is to turn off the "Merge Results" function in SolidWorks and use the "Feature Scope" function which allows the designer to choose the parts needed to merge with each other. We still create the seven parts as combinations of extrusion and other features needed as we did in the layout sketch method. The steps used to create the gearbox assembly are listed below:

1. Create the housing feature: after opening a new part, create the housing sketch shown in Figure 34.

\begin{tabular}{|c|c|c|}
\hline Figure 34 Gearbox housing sketch & Figure 35 Common cut feature sketch \\
\hline
\end{tabular}


Notice that Figure 34 is different from Figure 22. Extrude the sketch and perform other features to create the gearbox housing feature (same as shown in Figure 23).

2. Create the two large spur gears: create the gear sketch (same as shown in Figure 24). Extrude the sketch and perform other feature to create the gear feature (same as shown in Figure 25).

3. Create the two small spur gears: create the gear sketch (same as shown in Figure 26). Extrude the sketch and perform other feature to create the gear feature (same as shown in Figure 27).

4. Create the remaining components: the master model method allows the designer to create common cuts for in all existing components. This can be achieved by using the "feature scope" option available in SolidWorks during feature operations. As shown in Figures 35, we can create one sketch with two circles. We use the sketch to create an extrude cut as shown in Figure 36. We only cut the three features selected and shown under the "Feature Scope" pane shown. This option speeds up the modeling activities.

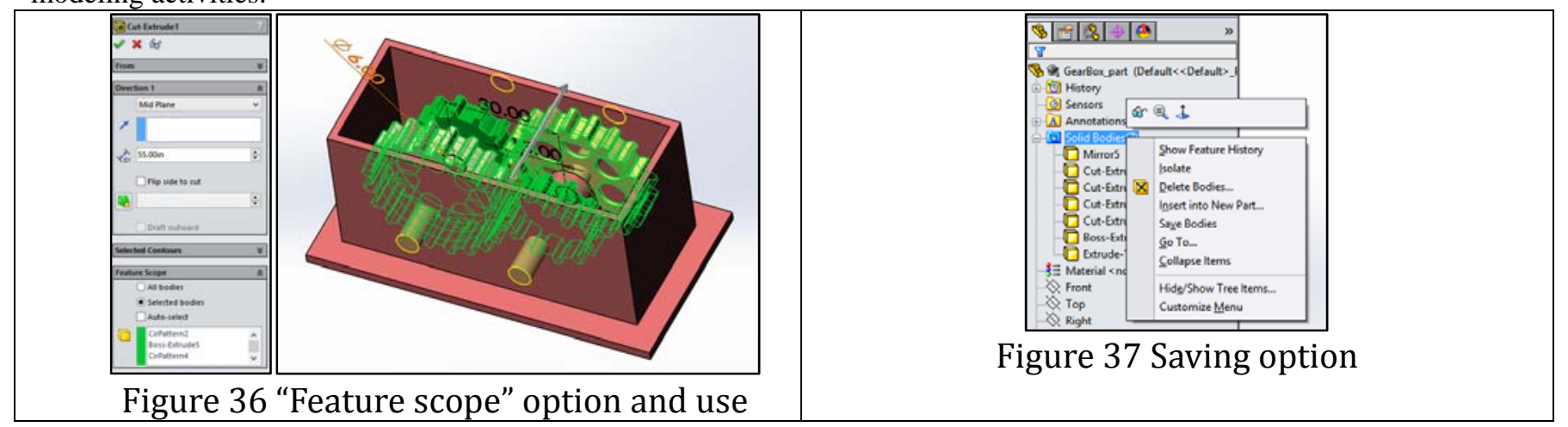

5. Create the shaft feature: create the shaft sketch (same as shown in Figure 28) and the shaft feature (same as shown in Figure 29).

6. Create the connector feature: create the connector sketch (same as shown in Figure 30) and the connector feature (same as shown in Figure 31).

7. Save the gearbox parts externally: As shown in Figure 37, right-click the Solid Bodies features node > Save Bodies.

The final gearbox assembly is the same as shown in Figures 32 and 33. Similar to the layout sketch method, the assembly, at this stage, does not have assembly mates between its seven individual parts. But they can be added if needed. The gearbox parts are shown assembled because they are created at their proper sketch locations.

As we proceed to complex assemblies such as a gearbox, we have observed that number of steps involved in creating the same assembly increases significantly in the layout sketch method as compared to the master model method. Also, the visualization and the relations between components becomes limited in the layout sketch method.

\section{ANALYSIS AND DISCUSSION}

We have presented three case studies to enable us to investigate the two methods commonly used in practice to create assemblies using the top-down approach: layout sketch and master model. The three case studies involve three assemblies: simple, intermediate, and complex. The four differentiating criteria of the three assembly models are the number of parts per assembly, the number of sketch planes required to create assembly parts, total number of feature required to create all assembly part, and the number and type of features required to create a given part. The pencil represents a simple assembly. The hinge represents an intermediate assembly. And the gearbox represents a complex assembly. Table 1 shows the first three criteria for each assembly. Table 2 shows the fourth criterion for each assembly.

During part and assembly creation we have discovered that the required number of sketch planes to create assembly parts is the most decisive criterion in determining the complexity level of creating assemblies using the top-down approach. This is because the layout sketch method in SolidWorks allows only one plane (front) for construction, thus severely limiting the modeling flexibility. Thus, we have classified the assemblies for our three case studies based on this criterion. 
Table 1. Assembly comparisons based on first three criteria

\begin{tabular}{|l|l|l|l|}
\hline \multirow{2}{*}{ Assembly } & \multicolumn{3}{|c|}{ Criteria } \\
\cline { 2 - 4 } & $\begin{array}{l}\text { Number } \\
\text { of parts }\end{array}$ & $\begin{array}{l}\text { Required number of } \\
\text { sketch planes to } \\
\text { create assembly parts }\end{array}$ & $\begin{array}{l}\text { Total } \\
\text { number of } \\
\text { assembly } \\
\text { features }\end{array}$ \\
\hline Pencil & 4 & 1 & 4 \\
\hline Hinge & 3 & $\begin{array}{l}3 \text { (1 ref plane, right, top } \\
\text { plane) }\end{array}$ & 7 \\
\hline Gearbox & 7 & $\begin{array}{l}\text { 5 (2 ref plane, front, top } \\
\text { right plane) }\end{array}$ & 23 \\
\hline
\end{tabular}

Table 2. Assembly comparisons based on fourth criterion

\begin{tabular}{|c|c|c|c|}
\hline Assembly & Part & Feature type & $\begin{array}{c}\text { Total } \\
\text { number of } \\
\text { features }\end{array}$ \\
\hline \multirow{4}{*}{ Pencil } & Body & Revolve & 1 \\
\hline & Lead & Revolve & 1 \\
\hline & Cap & Revolve & 1 \\
\hline & Eraser & Revolve & 1 \\
\hline \multirow{7}{*}{ Hinge } & \multirow{3}{*}{ Plate 1} & Extrude & \multirow{3}{*}{3} \\
\hline & & Extrude cut & \\
\hline & & Linear Pattern & \\
\hline & \multirow{3}{*}{ Plate 2} & Extrude & \multirow{3}{*}{3} \\
\hline & & Extrude cut & \\
\hline & & Linear Pattern & \\
\hline & Pin & Extrude & 1 \\
\hline \multirow{17}{*}{ Gear Box } & \multirow{3}{*}{ Housing } & Extrude (5) & \multirow{3}{*}{7} \\
\hline & & Extrude Cut & \\
\hline & & Mirror & \\
\hline & \multirow{5}{*}{ Gear 1} & Extrude & \multirow{5}{*}{6} \\
\hline & & $\begin{array}{l}\text { Reference } \\
\text { Geometry }\end{array}$ & \\
\hline & & Mirror & \\
\hline & & $\begin{array}{c}\text { Extrude Cut } \\
\text { (2) }\end{array}$ & \\
\hline & & $\begin{array}{c}\text { Circular } \\
\text { Pattern }\end{array}$ & \\
\hline & \multirow{5}{*}{ Gear 2} & Extrude & \multirow{5}{*}{6} \\
\hline & & $\begin{array}{l}\text { Reference } \\
\text { Geometry }\end{array}$ & \\
\hline & & Mirror & \\
\hline & & $\begin{array}{c}\text { Extrude Cut } \\
\text { (2) }\end{array}$ & \\
\hline & & $\begin{array}{c}\text { Circular } \\
\text { Pattern }\end{array}$ & \\
\hline & Gear 3 & Extrude & 1 \\
\hline & Gear 4 & Extrude & 1 \\
\hline & Shaft & Extrude & 1 \\
\hline & Connector & Extrude -Thin & 1 \\
\hline
\end{tabular}

With the above two tables in mind we offer the following metric summary: 
Table 3. Comparison metric

\begin{tabular}{|l|l|l|l|}
\hline Issue & Case study & $\begin{array}{l}\text { Layout } \\
\text { sketch }\end{array}$ & Master model \\
\hline \multirow{2}{*}{$\begin{array}{l}\text { Number of } \\
\text { steps }\end{array}$} & Pencil & 38 & 32 \\
\cline { 2 - 4 } & Hinge & 28 & 19 \\
\cline { 2 - 4 } $\begin{array}{l}\text { Limited use } \\
\text { of sketch } \\
\text { entities }\end{array}$ & All cases & 43 & 29 \\
\hline $\begin{array}{l}\text { Importing } \\
\text { external } \\
\text { blocks }\end{array}$ & All cases & $\begin{array}{l}\text { All instances } \\
\text { are linked } \\
\text { together }\end{array}$ & N/A \\
\hline $\begin{array}{l}\text { Sketch plane } \\
\text { orientation }\end{array}$ & All cases & $\begin{array}{l}\text { If not aligned } \\
\text { with layout } \\
\text { sketch plane } \\
\text { (Front), } \\
\text { feature } \\
\text { creation } \\
\text { becomes } \\
\text { difficult }\end{array}$ & $\begin{array}{l}\text { No } \\
\text { exist }\end{array}$ \\
\hline $\begin{array}{l}\text { Other } \\
\text { modeling } \\
\text { challenges }\end{array}$ & All cases & Too many & None \\
\hline
\end{tabular}

We elaborate Table 3 further as follows:

1. Number of steps: as shown in the table, the layout sketch method is cumbersome and require more steps that the master model method. This stems from two problems: (1) only one sketch plane (Front) is available regardless of feature complexity, and (2) the block concept limits multiple use of same entity to create multiple features.

2. Limited use of sketch entities: a block in the layout sketch method owns its entities exclusively, preventing their re-use in another block definition. If one sketch entity is needed for two blocks, we must create the entity twice. For example, if we create a centerline to create a revolve, we cannot use it again in another revolve. Such a limitation does not exist in the master model method.

3. Importing external blocks: if we use two instances of same external block into a layout sketch, any operation on one instance is applied automatically to the other. For example, we encountered this problem in creating the gearbox. One work around is to insert one instance, go back and rename the external block, then insert the second instance, thus separating the link between them.

4. Sketch plane orientation: the easiest case for the use of the layout sketch is when the feature sketch plane is aligned with the layout sketch plane, i.e. when both are front planes. If the two planes are orthogonal or oriented differently, it becomes a challenge to create the parts. For example, we faced this problem in the hinge creation. The master model method does not suffer from this problem.

5. Other modeling challenges: many other similar issues exist and are numerous to list here. For example, SolidWorks creates layout mates automatically. We had to delete some of these mates to define blocks from sketch. Entities

\section{CONCLUSION}

The layout sketch method is very limited, cumbersome, and time-consuming in SolidWorks. The master model method is much more versatile and powerful. However, we should mention that other CAD/CAM systems such as Creo have much better implementation of the layout sketch method.

\section{REFERENCES}

1. George Gustav Savii, Computer-Aided Design, In Encyclopedia of Information Systems, edited by Hossein Bidgoli, Elsevier, New York, 2003. p. 171-186

2. X.F Zha, H.J Du, J.H Qiu, Knowledge-based approach and system for assembly oriented design, Part I: the approach, Engineering Applications of Artificial Intelligence, 14 (1) February 2001, p. 61-75 
3. Arun Mathew C.S.P. Rao, (2010),"A CAD system for extraction of mating features in an assembly", Assembly Automation, Vol.30(2): p. $142-146$

4. Ibrahim Zeid. 2014. Mastering Solidworks (2nd ed.). Peachpit Press, Berkeley, CA, USA.

5. Ibrahim Zeid. 2004. Mastering Cad/Cam. McGraw-Hill, Inc., New York, NY, USA.

6. http://www.solidworks.com

7. http://www.ptc.com/product/creo

8. Sandberg, Marcus, Michael Kokkolaras, Jan-olov Aidanpaa, Ola Isaksson, Tobias Larsson. 2009. "A master-model approach to whole jet engine analysis and design optimization". WCSMO-8: 8th World Congress on Structural and Multidisciplinary Optimization.

9. Massimo Gallizio, Gian Paolo De Poli., Matteo Usseglio. 2006. “ Top dpwn can modeling methodology for low pressure turbine design” ICAS : $26^{\text {th }}$ international congress of the aeronautical sciences. 